\title{
The Survivin saga goes in vivo
}

John C. Reed

The Burnham Institute, 10901 N. Torrey Pines Road, La Jolla, California 92037, USA.

Phone: (858) 646-3140; Fax: (858) 646-3194; E-mail: jreed@burnham.org.

J. Clin. Invest. 108:965-969 (2001). DOI:10.1172/JCI200114123.

Survivin is a bifunctional protein that suppresses apoptosis and regulates cell division (1). This protein has garnered great interest as a potential drug target because its expression is among the most tumor-specific of all human gene products (2). While fetal tissues contain abundant Survivin mRNA and protein, most normal adult tissues do not. In contrast, the vast majority of tumors express Survivin protein at high levels, suggesting that reactivation of SURVIVIN gene expression occurs commonly in cancers (3).

The mechanisms by which Survivin regulates cell death and cell division are highly controversial. Indeed, even the premise that Survivin regulates apoptosis has been challenged, primarily on the grounds that Survivin homologues in yeast and Caenorbabditis elegans operate as regulators of cell division but not cell survival (reviewed in refs. 4, 5). Heretofore, the preponderance of evidence that mammalian Survivin suppresses apoptosis was based on overexpression of the protein in cultured cell lines. The absence of in vivo data on this point has now been remedied with a paper in this issue of the JCI by Altieri and colleagues (6). In an accompanying paper (7), the same investigators show how emerging knowledge about Survivin regulation might be use to develop a new gene therapy strategy for cancer.

Seeking to model events in tumors, Grossman et al. (6) placed the Survivin cDNA under the control of the keratin 14 (K14) promoter and generated transgenic mice that produce Survivin constitutively in epidermal keratinocytes. The skin is an easily accessible tissue that displays the full gamut of events relevant to tumorigenesis, including cell proliferation, differentiation, and death. Betting persons might have placed their wagers on seeing a defect in cell division or differentiation in K14-Survivin mice, but such was not the case. Instead, the ker- atinocytes of these Survivin-overexpressing mice demonstrated resistance to apoptosis, thus providing the first evidence that Survivin can suppress cell death in vivo. Specifically, overexpression of Survivin significantly reduced the numbers of apoptotic cells generated in the epidermis following exposure to ultraviolet-B (UVB) radiation (sunburn). While these data do not go as far as to establish a role for Survivin in homeostatic programmed cell death, they do at least indicate that the overexpression of Survivin (which is seen in many tumors) can result in an antiapoptotic state in vivo.

\section{Survivin and the IAPs}

Intuitively, it would seem that clues to the mechanism by which Survivin blocks apoptosis could be gleaned from work on other members of the same protein family, the IAPs (inhibitor-of-apoptosis proteins). IAPfamily members all contain at least one copy of a BIR domain, a zinc-binding fold. The presence of a BIR and evidence of apoptosis suppression upon overexpression in cells defines membership in the IAP family (reviewed in refs. 8, 9). Based on these criteria, humans contain eight IAP-family members: NAIP, cIAP1, cIAP2, XIAP, Ts-XIAP, ML-IAP, Apollon, and Survivin. Several IAPs from humans and Drosophila have been shown to directly bind and potently inhibit caspases, the cell death proteases responsible for apoptosis $(8,9)$. For example, the XIAP protein, which contains three BIR domains, binds and inhibits caspase- 9 through its third BIR domain (BIR3), while suppressing caspase- 3 and caspase-7 through a linker region located between BIR1 and BIR2 (10). Some reports have claimed Survivin directly suppresses caspase-3, but comparison of the $x$-ray crystallographic structures of Survivin with the XIAP(BIR2):caspase- 3 complex fails to suggest how Survivin could do this (11). In particu- lar, Survivin is lacking a long extension off the BIR domain that XIAP uses to occlude access to the active site of the caspase enzyme. More likely, Survivin may bind caspase-9 (12), since its BIR domain is closely related in threedimensional structure to the XIAP BIR3 domain, which binds and inhibits this enzyme in vitro (13). However, direct evidence that purified Survivin can directly bind and inhibit purified caspase-9 is still lacking.

One potential explanation for difficulties in demonstrating direct effects of Survivin on caspase- 9 came to light last year, when the Altieri group reported that Survivin phosphorylation on threonine 34 (T34) is necessary for association of Survivin with processed caspase-9 (12). Crucially, a mutant of Survivin in which T34 is mutated to nonphosphorylatable alanine (T34A) disrupts cell division and induces apoptosis, presumably by competing with endogenous Survivin for access to kinases, thereby preventing the phosphorylation of the wildtype protein. Although these data argue for a critical role for phosphorylation in Survivin function, it is puzzling that the threonine residue in question resides near an acidic patch of amino acids on the surface of the Survivin molecule (13); the known 3D structures of caspases fail to suggest a reason why phosphorylation at T34, which would only add to the acidic character of this region, should promote interaction with caspase-9.

The question remains as to whether Survivin directly binds and inhibits caspase-9. It is entirely possible that the association of these proteins is indirect, requiring intermediate proteins that promote association of caspase-9 and Survivin with the same protein complex. Interestingly, Survivin has recently been shown to bind SMAC (Diablo), a proapoptotic protein that binds IAPs and prevents them from suppressing caspases, via a 
Antiapoptotic Mechanism of Survivin?

\title{
Survivin $\longrightarrow$ SMAC $\longrightarrow$ XIAP $\longrightarrow$ Caspases $\longrightarrow$ Apoptosis
}

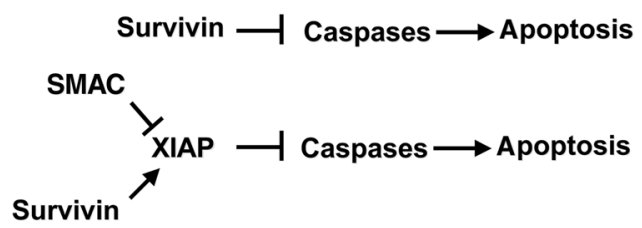

\begin{abstract}
Figure 1
Models for Survivin function. Several models are proposed that might explain how Survivin directly or indirectly inhibits caspases. Survivin might directly bind and inhibit caspase, analogous to IAPs, such as XIAP (top). Survivin might sequester SMAC, thus protecting IAPs from this inhibitory protein (middle). Finally, Survivin might somehow enhance the function of IAPs, having a function opposite to SMAC (bottom).
\end{abstract}

mechanism highly similar to that employed by analogous proteins $(\mathrm{Rpr}$, Hid, Grim) in Drosophila $(14,15)$. Thus, Survivin theoretically could bind and counteract SMAC, making it an inhibitor of an inhibitor of an inhibitor (Figure 1), rather than a direct suppressor of caspases. In this model, Survivin could protect other IAPs such as XIAP from inhibition by SMAC, allowing them to maintain their suppression of caspases. Comparisons with Detrin, the Drosophila homolog of Survivin, and investigation of whether it binds the fly counterparts of SMAC (Rpr, Hid, Grim), could be illuminating, given the striking parallels between flies and humans with respect to apoptosis regulation by IAPs (reviewed in ref. 16). The convincing dominant-negative effect of Survivin T34 phosphorylation must be taken into account in judging any proposed model of this protein's regulatory mechanisms (Figure 1).

\section{Intrinsic and extrinsic routes to apoptosis}

Functional analysis of Survivin by gene transfection has provided insights into the possible mechanism by which Survivin blocks apoptosis, and some of these observations are consistent with the hypothesis of an effect on caspase-9. At least two major pathways for caspase activation and apoptosis have been defined (17). In one, the so-called "extrinsic" pathway, signals from TNF-family receptors are transduced via a network of adapter proteins, resulting in activation of the upstream initiator protease, caspase-8. In contrast, the "intrinsic" pathway involves mitochondria, which release cytochrome $c$ in response to various noxious stimuli, including DNA damage, with cytochrome $c$ binding and activating Apaf1, which in turn binds and activates caspase-9. Caspase- 8 and caspase- 9 then cleave and activate various downstream effector caspases, with caspase- 3 representing the most commonly identified next-link in this protease cascade (Figure 2). Cultured keratinocytes derived from K14-Survivin mice are resistant to apoptosis induced by UVB, a stimulator of the intrinsic pathway, but sensitive to apoptosis induced by TNF (6). Similarly, in their accompanying paper, Mesri et al. (7) show that expression of the Survivin(T34A) dominant-negative mutant in cultured tumor lines leads to depletion of pro-caspase-9, consistent with the activation of the intrinsic pathway. In addition, apoptosis induced by Survivin(T34A) can be blocked by coexpressing a dominant-negative form of caspase- 9 but not caspase-8 (12).

These correlative data suggest that Survivin controls the mitochondriadependent pathway for apoptosis, but other findings paint a more confusing picture. For example, in contrast to Survivin overexpressing keratinocytes derived from K14-Survivin mice, endothelial cells expressing high levels of Survivin as a result of recombinant adenovirus transduction are protected from TNF-induced apoptosis (18). Potentially, this discrepancy can be explained by cross-talk between the extrinsic and intrinsic pathways. The proapoptotic Bcl-2 family protein, Bid, provides one example of a cross-talk molecule, but others may also contribute (reviewed in ref. 19). In some cell types, ligation of TNF-family death receptors results in only small amounts of caspase- 8 activation, and successful execution of apoptosis requires recruitment of the mitochondria pathway - often via caspase8-mediated cleavage and activation of Bid, which targets mitochondrial membranes and triggers cytochrome $c$ release (Figure 2) (20).

Also confusing is the observation that overexpression of Survivin(T34A) induces cytochrome $c$ release. If Survivin operates solely as an antagonist of caspase-9, one would not necessarily expect the act of overexpressing Survivin(T34A) by itself to cause mitochondria to dump their contents, since cytochrome $c$ release occurs upstream of caspase- 9 in the intrinsic pathway (Figure 2). This observation raises the possibility that apoptosis induction by Survivin(T34A) is secondary to some event impinging upon the intrinsic pathway upstream of or at the level of mitochondria - perhaps triggered as a result of the disruption in cell division that occurs in cells expressing this Survivin mutant (12). It should be noted, however, that a feedback amplification loop exists in which caspases activated downstream of mitochondria circle back and cause more mitochondria to expel their cytochrome $c$ (Figure 2). Such a response could account for why cytochrome $c$ release is induced by Survivin(T34A), but even this provides only a partial explanation, since it does not account for the initial activation of caspase-9. Determining the mechanism of Survivin(T34A)-induced apoptosis is not just an academic exercise, because it has implications for selecting patients who might benefit eventually from Survivin-based therapies, and avoiding those whose tumors contain blocks in apoptosis pathways that would prevent a response to Sur$\operatorname{vivin}(\mathrm{T} 34 \mathrm{~A})$

\section{Survivin and cell division}

Just as cytochrome $c$ release may arise as an indirect consequence of expressing the nonphosphorylatable T34A mutant of Survivin, the cytoprotection achieved by overexpressing wild-type Survivin might also be secondary to its effects on some other cellular process. Interestingly, Survivin is required for 
proper execution of mitosis and cell division, and thus defects in Survivin could trigger a cell cycle checkpoint response linking cell suicide to errors in chromosome segregation (reviewed in ref. 5). The promoter of the SURVIVIN gene of mammals contains several elements typical of cell cycle-regulated genes and SURVIVIN is regulated in a highly cell cycle-dependent manner in normal cells such as peripheral blood lymphocytes and endothelial cells (21). Even in cultured tumor cell lines examined, Survivin mRNA and protein accumulate selectively during or around the time of mitosis (reviewed in refs. 4, 5). Moreover, Survivin localizes to the mitotic spindle apparatus, including possibly mitotic microtubules, and is found associated with the last structure holding the two daughter cells together (the midbody) at the end of telophase, just before splitting into two separate cells.

Consistent with these characteristics, interference with Survivin function or expression in cultured tumor cell lines by expression of dominant-negative mutants or by antisense-mediated reductions in SURVIVIN expression has been associated with supernumerary centrosomes, aberrant mitosis, and defective cytokinesis, with cells becoming polyploid and multinucleated. Homozygous disruption of both alleles of the Survivin gene in mouse embryonic stem cells results in embryonic lethality at day 4-5 due to chromosome segregation problems and failed cytokinesis (22). Moreover, knockouts of putative Survivin homologues in fission yeast (bir-1) and budding yeast (BIR-1), and knock-downs of a Survivin-like gene (bir-1) in C. elegans embryos, cause various defects in centrosome formation, mitotic spindle assembly, chromosome segregation, and cytokinesis (reviewed in ref. 5). Thus, loss-of-function mutations or interference with endogenous function or expression of Survivin and its homologues in lower organisms clearly results in defects in late steps of cell division. Often these defects in cell division are followed by cell death, but it could be argued that most anything that disrupts chromosome segregation could result secondarily in cell demise. How Survivin and its homologues assist in chromosome segregation and cytokinesis is unknown, but evidence has been presented support- ing an adapter protein-like role for Survivin homologues in targeting Ipl1/Aurora-family protein kinases to proper locations (and hence to proper substrates) on mitotic chromosomes and the mitotic spindle apparatus.

The apparent requirement for Survivin for normal cell division suggests that overexpression of this protein (as is seen, for example, in many tumors) could perturb normal cell cycle control. Such a response might well account for Survivin's ability to block apoptosis, given the intimate connection between cell cycle checkpoints and the commitment phase of apoptosis. At least in the epidermis of K14-Survivin mice, rates of cell division seem to be normal, even when challenged by UV irradiation or treated with the tumor promoter, PMA (6). However, the apoptotic stimulus for which Survivin-mediated protection was demonstrated was UVB irradiation. This treatment damages DNA and induces a p53-dependent checkpoint that can lead to both cell cycle arrest and apoptosis.

To explore the possible connection between cell death suppression and Survivin's effects on cell division, it would be useful to test the cellular response to apoptotic stimuli that are cell cycle-independent (growth factor deprivation; cell detachment [anoikis]; staurosporine) on keratinocytes from the K14-Survivin mice, and even better if the experiments could be performed on quiescent, noncycling cells. The original description of Survivin showed that forced overexpression of this protein could slow cell death induced by IL-3 withdrawal from a factor-dependent hematopoietic cell line that undergoes $\mathrm{G}_{0}$ arrest prior to dying (3). Hence, Survivin can evidently suppress cell death in a cell cycle-independent fashion. However, while overexpression of Survivin protects

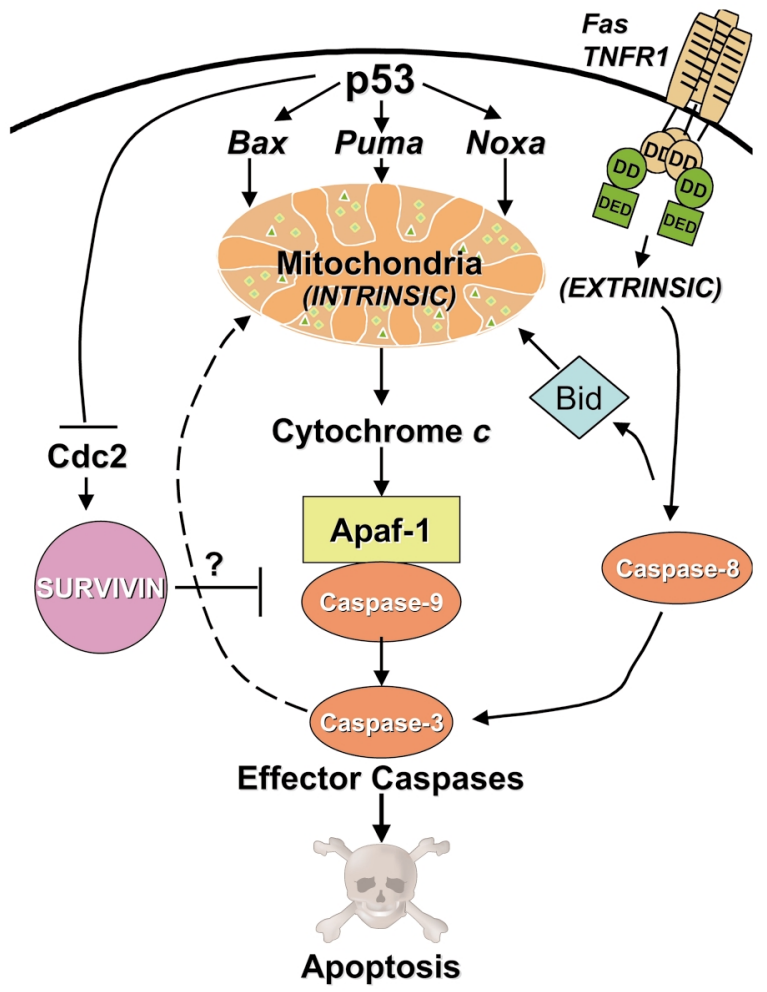

\section{Figure 2}

Two routes to apoptosis. In the "intrinsic" cell death pathway, various upstream stimuli, such as activation of $\mathrm{p} 53$, induce expression or activation of proapoptotic Bcl-2 family proteins (e.g., Bax, Puma, Noxa) that converge on mitochondria and induce cytochrome $c$ release. In the cytosol, cytochrome $c$ binds Apaf1, which activates caspase-9. In the "extrinsic" pathway, TNF-family receptors trigger caspase- 8 activation. Active caspase- 8 and caspase- 9 cleave and activate caspase- 3 and possibly other downstream effector caspases. Caspase- 8 can also cleave and activate Bid, resulting in mitochondrial release of cytochrome $c$. Once activated, downstream caspases can also damage mitochondria, representing a potential amplification mechanism. The putative point of Survivin intervention at caspase- 9 is shown. Cdc2 may be required for apoptosis suppression by Survivin. Expression of Cdc2 can be repressed by $\mathrm{p} 53$ in $\mathrm{G}_{2}$-phase. DD, death domain; DED, death effector domain. 
keratinocytes from apoptosis induced by UVB, the basal rates of cell death in the epidermis are not obviously affected, and the thickness of the epidermis was not perturbed, suggesting that programmed cell death associated with normal differentiation occurred unabated. Perhaps this observation is not surprising, given that mice lacking caspase-9 (the presumptive target of Survivin) have normal skin, but the point stands that Survivin probably does not deregulate all cell death pathways. More work is clearly needed to define Survivin's niche in apoptosis regulation more precisely.

\section{Regulation of Survivin by phosphorylation}

The requirement for T34 phosphorylation of Survivin has important implications for understanding Survivin's potential limitations in cell death regulation. At present, the only kinase recognized to phosphorylate Survivin is Cdc2, a cyclin-dependent kinase that is only active during certain points in the cell cycle (12). Consistent with a role for $\mathrm{Cdc} 2$ in Survivin phosphorylation, kinasedead mutants of Cdc2 prevent this modification, and the enzyme can be coimmunoprecipitated with Survivin in cells that have been synchronized in mitosis. Moreover, $\mathrm{Cdc} 2$ colocalizes with Survivin on mitotic spindle microtubules and midbodies. Though other members of the Cdc2 family of kinases may also associate with Survivin (23), they too are active only during certain cell cycle transitions, and thus the activity of Survivin as an apoptosis-blocking protein is likely restricted to dividing cells - and possibly to tumors where deregulated constitutive activation of Cdc2-family kinases occurs. These and other observations imply a narrow rather than generic role for Survivin in apoptosis suppression, possibly linked to cell cycle checkpoints where cells that make mistakes in chromosome segregation or cytokinesis may fail to phosphorylate Survivin and thus perish, as mentioned above. Thus Survivin may be protective in keratinocytes because UV-damaged cells arrest in phases of the cell cycle where $\mathrm{Cdc} 2$ normally becomes active. Time-lapsed microphotography of these cells would likely provide insights into this question.
However, it is provocative that, in contrast to normal cells where SURVIVIN gene expression is restricted to the $\mathrm{G}_{2} / \mathrm{M}$ phase of the cell cycle, in tumors immunohistochemical analysis typically reveals homogeneous immunostaining of nearly all the malignant cells, suggesting deregulated expression. Moreover, the location of the Survivin protein is abnormal in tumors in vivo, with Survivin present diffusely throughout the cytosol of most tumor cells. Indeed, in cultured keratinocytes derived from K14-Survivin mice, Survivin protein is found predominantly in the cytosol and is excluded from the nucleus (6). These observations suggest that Survivin blocks apoptosis within the cytosol, where caspases are predominantly located.

The paper by Grossman et al. (6) reports the additional provocative observation that loss of a $p 53$ allele reduces UVB-induced keratinocyte apoptosis further than is seen with K14Survivin overexpression alone. By mating K14-Survivin mice with $p 53$ knockouts, the authors observed that heterozygosity for $p 53$ together with Survivin overexpression enhances cell survival following UVB treatment, reducing the number of sunburned (apoptotic) cells from about 7 per centimeter in the K14-Survivin mice to about 4.5 per centimeter in the K14-Survivin $/ p 53^{+/-}$mice and reducing the percentage of apoptosis in culture from $\sim 4.5 \%$ to $\sim 1.5 \%$. This modest effect, which has so far been demonstrated with only one K14-Survivin founder line and on a single genetic background, is insufficient to suggest genetic complementation between $p 53$ loss and Survivin overexpression. But it could be that p53 and Survivin operate in the same pathway in regulating apoptosis, consistent with evidence that caspase-9 is a critical downstream component of the p53-dependent tumor suppressor pathway (24). Nevertheless, the findings raise the possibility of a functional interaction of $\mathrm{p} 53$ with Survivin, which suggests many possibilities, including perhaps regulation by $\mathrm{p} 53$ of the expression of Survivin or a Survivin-binding protein. In this regard, p53-dependent repression of $\mathrm{Cdc} 2$ expression has been demonstrated during $\mathrm{G}_{2}$-arrest (25), suggesting the testable hypothesis that p53 loss is associated with greater phosphorylation of Survivin and thus more complete suppression of apoptosis.

\section{Strategies for counteracting Survivin in tumors}

The 3D structure of Survivin fails to suggest any obvious opportunities for directly binding small-molecule drugs to this protein. While experiments with antisense oligonucleotides and cultured cancer cells have provided proof-of-concept evidence that loss of Survivin expression can disrupt cell division and trigger apoptosis $(26,27)$, these findings have yet to be extended into preclinical animals models. The striking effects of the T34A mutant on apoptosis and cell division in tumor cell lines suggest the possibility of exploiting this Survivin mutant in gene therapy strategies for cancer. Earlier data, based on a melanoma xenograft model, showed that induction of the mutant Survivin(T34A) protein suppressed tumor growth in vivo (28). In this issue of the JCI, Mesri et al. have now extended those findings into more clinically germane xenograft models, using a breast cancer line and delivering a recombinant adenovirus encoding Survivin(T34A) into subcutaneous tumor nodules or into tumor-bearing peritoneum (7). The authors observed striking antitumor activity, comparable to that seen with the drug paclitaxel $\left(\operatorname{Taxol}^{\circledR}\right)$. In vitro, Survivin(T34A) adenovirus inhibited proliferation and induced apoptosis in five of five tumor lines tested, while normal fibroblasts, smooth muscle cells, and endothelial cells were not affected (7). Why normal cells are not harmed by Survivin(T34A) under these conditions is unclear. Endothelial cells, for example, upregulate their Survivin expression 16fold after stimulation with VEGF (18), which suggests they employ Survivin for something. The Survivin(T34) used in these experiments was fused with the green fluorescent protein (GFP), which can have toxicity in some types of cells but not others (29). Could it be that the combination of GFP plus Survivin(T34) is selectively toxic to transformed but not normal cells? Alternatively, tumor cells may simply be more dependent on Survivin than are healthy cell types.

Knowledge about mechanisms of Survivin regulation suggests that we may already have drugs that indirectly inactivate this protein. For example, Cdc2 kinase inhibitors such as flavopiridol are already in clinical trials and would be expected to inactivate Survivin by preventing its phosphorylation. Also, a domain in Survivin has 
been identified that seems to interact with microtubules, and mutations in this domain abolish Survivin's function (30), so perhaps antimicrotubule drugs such as vincristine and vinblastine are indirect inhibitors of Survivin.

While therapeutic uses of Survivin remain to be defined, its potential utility in early diagnosis of cancer is incontrovertible. A question that ought to be explored is whether Survivin is released into blood or accumulates in urine of patients with cancer, providing a basis for novel diagnostics for early detection of cancer, prognostic markers for aiding patient management, or endpoints for monitoring disease during and after therapy.

\section{Acknowledgments}

I thank R. Abraham, T. Hunter, J. Ashkenas, and G. Salvesen for helpful discussions, and R. Cornell for manuscript and figure preparation.

1. Altieri, D.C., Marchisio, P.C., and Marchisio, P.C. 1999. Survivin apoptosis: an interloper between cell death and cell proliferation in cancer. $L a b$. Invest. 79:1327-1333.

2. Velculescu, V.E., et al. 1999. Analysis of human transcriptomes. Nat. Genet. 23:387-388.

3. Ambrosini, G., Adida, C., and Altieri, D. 1997. A novel anti-apoptosis gene, survivin, expressed in cancer and lymphoma. Nat. Med. 3:917-921.

4. Reed, J.C., and Reed, S.I. 1999. Survivin' cell-sep- aration anxiety. Nat. Cell Biol. 1:E199-E200.

5. Reed, J.C., and Bischoff, J.R. 2000. BIRinging chromosomes through cell division - and survivin the experience. Cell. 102:545-548.

6. Grossman, D., et al. 2001. Transgenic expression of survivin in keratinocytes counteracts UVBinduced apoptosis and cooperates with loss of p53. J. Clin. Invest. 108:991-999.

7. Mesri, M., Wall, N.R., Li, J., Kim, R.W., and Altieri, D.C. 2001. Cancer gene therapy using a survivin mutant adenovirus. J. Clin. Invest. 108:981-990.

8. Deveraux, Q., and Reed, J. 1999. IAP family proteins: suppressors of apoptosis. Genes Dev. 13:239-252.

9. Miller, L. 1999. An exegesis of IAPs: salvation and surprises from BIR motifs. Trends Cell Biol. 9:323-328.

10. Sun, C., et al. 1999. NMR structure and mutagenesis of the inhibitor-of-apoptosis protein XIAP. Nature. 401:818-821.

11. Riedl, S.J., et al. 2001. Structural basis for the inhibition of caspase-3 by XIAP. Cell. 104:791-800.

12. O'Connor, D.S., et al. 2000. Regulation of apoptosis at cell division by $\mathrm{p} 34^{\text {cdc2 }}$ phosphorylation of survivin. Proc. Natl. Acad. Sci. USA. 97:13103-13107.

13. Shi, Y. 2000. Survivin structure: crystal unclear. Nat. Struct. Biol. 7:620-623.

14. Du, C., Fang, M., Li, Y., Li, L., and Wang, X. 2000. Smac, a mitochondrial protein that promotes cytochrome c-dependent caspase activation by eliminating IAP inhibition. Cell. 102:33-42.

15. Verhagen, A.M., et al. 2000. Identification of DIABLO, a mammalian protein that promotes apoptosis by binding to and antagonizing IAP proteins. Cell. 102:43-53.

16. Abrams, J. 1999. An emerging blueprint for apoptosis in drosophila. Trends Cell Biol. 9:435-440.

17. Cryns, V., and Yuan, Y. 1999. Proteases to die for Genes. Dev. 12:1551-1570.

18. O'Connor, D.S., et al. 2000. Control of apoptosis during angiogenesis by survivin expression in endothelial cells. Am. J. Pathol. 156:393-398.

19. Reed, J.C. 2000. Mechanisms of apoptosis. Am. J. Pathol. 157:1415-1430.

20. Huang, D.C., and Strasser, A. 2000. BH3-only proteins: essential initiators of apoptotic cell death. Cell. 103:839-842.

21. Li, F., et al. 1998. Control of apoptosis and mitotic spindle checkpoint by survivin. Nature. 396:580-587.

22. Uren, A.G., et al. 2000. Survivin and the inner centromere protein INCENP show similar cell-cycle localization and gene knockout phenotype. Cur Biol. 10:1319-1328.

23. Suzuki, A., et al. 2000. Survivin initiates cell cycle entry by the competitive interaction with Cdk4/p16 $6^{\mathrm{INK} 4 \mathrm{a}}$ and Cdk2/cyclin E complex activation. Oncogene. 19:3225-3234.

24. Soengas, M., et al. 1999. Apaf-1 and caspase-9 in p53-dependent apoptosis and tumor inhibition. Science. 284:156-159.

25. Passalaris, T.M., Benanti, J.A., Gewin, L., Kiyono, T., and Galloway, D.A. 1999. The $\mathrm{G}_{2}$ checkpoint is maintained by redundant pathways. Mol. Cell. Biol. 19:5872-5881.

26. Chen, J., et al. 2000. Down-regulation of survivin by antisense oligonucleotides increases apoptosis, inhibits cytokinesis and anchorage-independent growth. Neoplasia. 2:235-241.

27. Olie, R.A., et al. 2000. A novel antisense oligonucleotide targeting survivin expression induces apoptosis and sensitizes lung cancer cells to chemotherapy. Cancer Res. 60:2805-2809.

28. Grossman, D., Kim, P.J., Schechner, J.S., and Altieri, D.C. 2001. Inhibition of melanoma tumor growth in vivo by survivin targeting. Proc. Natl. Acad. Sci. USA. 98:635-640.

29. Martinez-Serrano, A., Villa, A., Navarro, B., Rubio, F.J., and Bueno, C. 2000. Human neural progenitor cells: better blue than green? Nat. Med. 6:483-484.

30. Li, F., et al. 1999. Pleiotropic cell-division defects and apoptosis induced by interference with survivin function. Nat. Cell Biol. 1:461-466. 